\title{
Comparison of Phenotypic Methods of Detection of Carbapenamases in a Tertiary Care Hospital, South India
}

\author{
M. Uma Maheswari* \\ Department of Microbiology, PSGIMSR, Coimbatore, India \\ *Corresponding author
}

\section{A B S T R A C T}

\begin{tabular}{|l|}
\hline Ke y w o r d s \\
Carbapenamase, \\
Carba Np test \\
\hline Article Info \\
\hline $\begin{array}{l}\text { Accepted: } \\
\text { 04 November } 2020 \\
\text { Available Online: } \\
\text { 10 December } 2020\end{array}$ \\
\hline
\end{tabular}

\begin{abstract}
Enterobacteriaceae are the most frequently isolated organisms from clinical specimens. Among them Escherichia coli and Klebsiella pneumoniae cause a wide variety of clinical infections. With the emergence of ESBLs, carbapenems became the drug of choice. During the past decade, there has been an emergence of carbapenems-resistant Enterobacteriaceae that produce carbapenamases. So detection of carbapenamase producing Enterobacteriaceae has become mandatory for effective treatment. Hundred isolates of Meropenam resistant Escherichia coli and Klebsiella pneumoniae were selected. Modified Hodge test, double disc synergy test, combined disc or Disc enhancement test and Carba $\mathrm{Np}$ Test were done for these resistant isolates among them. The sensitivity and specificity of Carba Np test was $100 \%$ and better than Modified Hodge test, combined disc test and double disc synergy test. Thus carba Np test is more effective and easily adoptable method used to identify the carbapenamase producing organisms.
\end{abstract}

\section{Introduction}

Gram negative bacteria belonging to the family of Enterobacteriaceae are the most frequently isolated organisms from clinical specimens. They are widely dispersed in soil, water, plants, they cause a wide variety of clinical infections. Treatment of these infections depends on the site and severity. Resistance produced by these organisms pose a great difficulty in treatment and hence detection of these resistance producing organisms becomes mandatory.

As the name indicates enterobacteriaceae is also present in intestinal tract of human and animals. The members of this family of Enterobacteriaceae exhibit general morphological and biochemical similarities.

Within the family they exhibit wide biochemical and antigenic heterogeneity. Enterobacteriaceae are inhabitants of wide variety of niches, including the human, animal gastrointestinal tract and various other environmental sites.

The family Enterobacteriaceae includes a large number and diversity of genera. Escherichia coli causes urinary tract infections, cystitis, bacteremia, neonatal meningitis, diarrhea and dysentery. Klebsiella 
causes a wide variety of nosocomial infections of respiratory tract, urinary tract, and other normally sterile sites. They most frequently infect hospitalized and seriously debilitated patients. ${ }^{1}$

Treatment of Escherichia coli infections depend on the site and severity of the infection. Escherichia coli are treated with Ampicillin, third generation cephalosporins like ceftriazone. Doxycycline, TMP/SMZ, fluroquinolones.

Klebsiella infections are intrinsically resistant to Ampicillin, Ticarcillin and nitrofurantoin is poorly active against them. In Severely ill patients with Klebsiella infections, the treatment options include third generation cephalosporins, quinolones and amino glycosides $^{2}$.

Escherichia coli and Klebsiella initially responded to betalactam antibiotic but there was a dramatic increase in resistance to betalactam antibiotics due to beta lactamases produced by them.

With the emergence of ESBLs, carbapenems became the drug of choice. During the past decade, there has been an emergence of carbapenems-resistant Enterobacteriaceae hat produced carbapenamases, enzymes that efficiently hydrolyze carbapenems, as well as most $\beta$-lactam drugs ${ }^{3}$.

\section{Materials and Methods}

After Ethical clearance from the institution, the study was conducted between April 2014 August 2015. Out of 2042 Escherichia coli and 1192 samples of Klebsiella pneumoniae isolated from clinical samples, 560 samples of Klebsiella pneumoniae (47\%) and 148 isolates of Escherichia coli (7\%) were resistant to carbapenems (Meropenam) by disc diffusion method. Hundred isolates of carbapenem resistant Escherichia coli and Klebsiella pneumoniae were selected randomly for comparison of phenotypic methods like.

1. Modified Hodge test

2. Double disc synergy test

3. Combined disc or Disc enhancement test

4. Carba Np Test

\section{Results and Discussion}

It was observed that $42 \%$ were positive by Double disc diffusion test, $75 \%$ were positive by Combination disc test, $90 \%$ were positive by Modified Hodge test and 98\% were positive by Carba Np test. The sensitivity and specificity of Carba Np test is $100 \%$ and is better than Modified Hodge test (sensitivity $91 \%$ specificity $100 \%$ ), Combined disc test (sensitivity $92 \%$ and specificity $50 \%$ ) and double disc synergy test (sensitivity $42 \%$ and specificity 50\%) (Fig. 1-3 and Table 1).

Table.1

\begin{tabular}{|l|l|c|c|c|c|}
\hline S.No & Name of the test & Positives & Negatives & Sensitivity & Specificity \\
\hline $\mathbf{1}$ & Double disc synergy test & 42 & 58 & 42 & 50 \\
\hline $\mathbf{2}$ & Combination disc test & 75 & 25 & 92 & 50 \\
\hline $\mathbf{3}$ & Modified Hodge test & 90 & 10 & 91 & 100 \\
\hline $\mathbf{4}$ & Carba NP test & 98 & 2 & 100 & 100 \\
\hline
\end{tabular}


Fig.1 Double disc synergy test and combination disc test

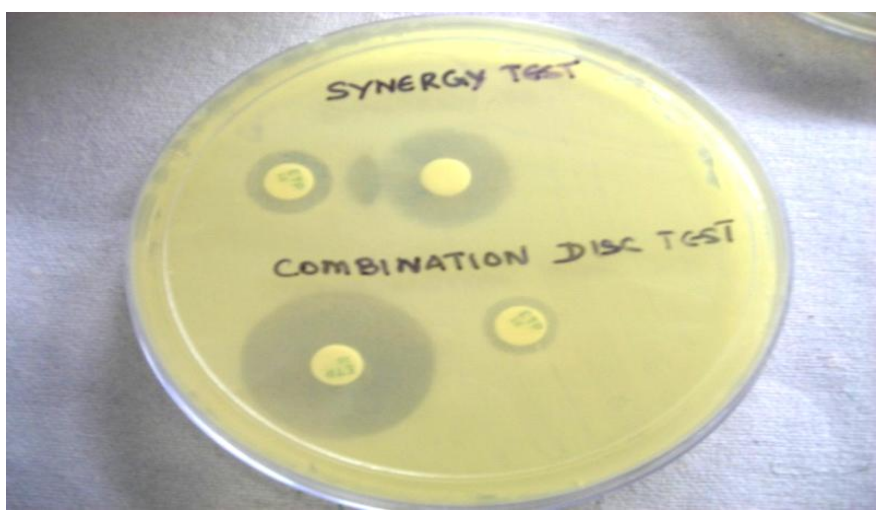

Fig.2 Modified Hodge test

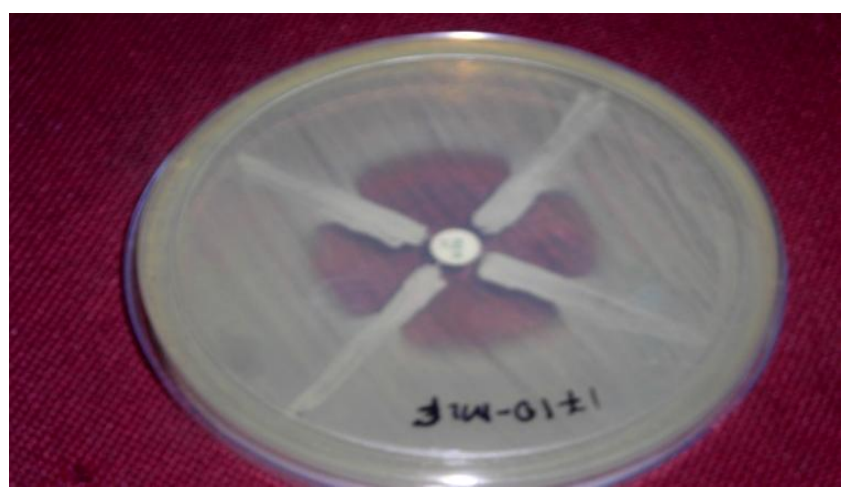

Fig.3 Carba NP test

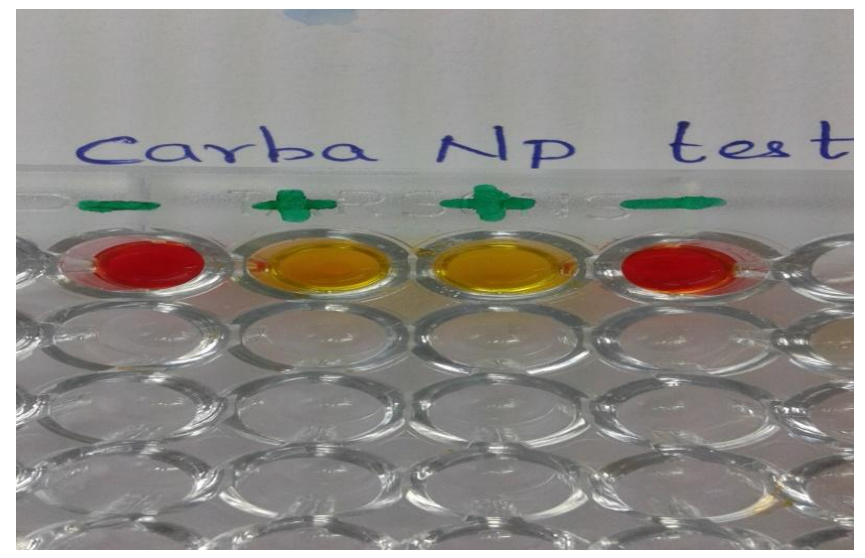

Enterobacteriaceae are inhabitants of wide variety of niches, including the human, animal gastrointestinal tract and various other environmental sites. The family Enterobacteriaceae includes a large number and diversity of genera. Escherichia coli and Klebsiella pneumoniae cause sepsis, endotoxic shock, urinary tract infections, meningitis in neonates and pneumonia in immune compromised They are normally 
treated based on the antibiotic sensitivity, site of infection and co morbidity condition. Escherichia coli and Klebsiella infections initially responded to betalactam antibiotics, but there was a dramatic increase in resistance to beta lactam antibiotics due to betalactamases produced by them. This resistance was superseded by use of second and third generation cephalosporins and combination of betalactam antibiotics with betalactamases inhibitors. Further with the emergence of ESBLs and Amp C resistance, carbapenems became the drug of choice. There has been a rapid increase in carbapenems resistance in Enterobacteriaceae in the recent years. The carbapenems resistance may be due to carbapenamases production, Efflux pump and Amp C enzyme production with membrane impermeability.

The various methods of carbapenem resistance detection are double-disc synergy, Combined disc, Modified Hodge and Carba Np test.

Double disc synergy test was done with Ertapenem, ceftazidime and disc with EDTA in between. Any distortion of zone towards EDTA disc was considered positive. Combination disc test was done with Ertapenem and Ertapenem with EDTA. An increase in zone diameter with combination disc of more than or equal to $5 \mathrm{~mm}$ was considered as positive. Clover leaf pattern on the Muller Hinton plate with in a lawn ATCC Escherichia coli and test strain streaked perpendicular to it indicates positivity..

Carba Np test is found to be the most specific and sensitive method, one caliberated loop of test organism was taken from a MHA plate. The colony was suspended in protein extraction lysis buffer, vortexed for 1minute, incubated at room temperature for 30 minutes and centrifuged at $10,000 \mathrm{rpm}$ for 5 minutes. $30 \mathrm{ul}$ of supernatant of centrifuged bacterial isolate was added to $100 \mathrm{ul}$ of a $1 \mathrm{ml}$ solution made of 3mg Imipenem monohydrate, $\mathrm{pH} 7.8$, phenol red solution and $0.1 \mathrm{mmol} / 1 \mathrm{Znso}_{4}$ and Incubated at $37^{\circ} \mathrm{c}$ for $2 \mathrm{hrs}$. The colour of wells changed from Red to yellow in all the test strains producing carbapenemases. The colour remained red for the negative strains.

In our study also we found that Carba $\mathrm{Np}$ test is most specific (100\%) and sensitive (100\%) in detecting carbapenamases and it was better than Modified Hodge test (sensitivity 91\% specificity 100\%),Combination disc test (sensitivity $92 \%$ and specificity $50 \%$ ) and Double disc synergy test (sensitivity $42 \%$ and specificity 50\%).

In another study on comparison of various phenotypic tests (DDST, CDT, MHT). Modified Hodge test was comparatively more specific and sensitive than Double disc synergy test and combination disc test ${ }^{4}$. Amjad et al observed carbapenamases in 69\% of isolates (Escherichia coli, Klebsiella pneumoniae, Pseudomonas aeruginosa, Acinetobacter baumanii, Citrobacter diversus and Enterobacter agglomerans) by Modified Hodge test ${ }^{5}$.

In a study on comparison of carbapenemase detection using a novel chromogenic rapid test-the Carba NP test and the modified Hodge test (MHT). Sensitivities were found to be comparable (CNP, $100 \%$, versus MHT, 98\%; $\mathrm{P}$ 0.08), but Carba $\mathrm{Np}$ was more specific $(100 \%$ versus $80 \%$; $\mathrm{P}<0.0001)$ and faster ${ }^{6}$.

In a similar study Carba NP test detected all carbapenemase producers regardless of the type of the carbapenemase and of their mucoid phenotype with $100 \%$ sensitivity and $100 \%$ specificity ${ }^{7}$.

Most of Carbapenem resistant strains were from urine samples (47\%). A similar study 
showed $57.89 \%$ carbapenamases producing Klebsiella pneumoniae in urine samples ${ }^{4}$. Khanduri et al., detected in his study that among 50 isolates of carbapenem resistant Enterobacteriaceae $44 \%$ were Klebsiella pneumoniae and $20 \%$ were Escherichia coli ${ }^{8}$

The various methods of carbapenem resistance detection are double-disc synergy, Combined disc, Modified Hodge and Carba Np test.

Double disc synergy test was done with Ertapenem, ceftazidime and disc with EDTA in between. Any distortion of zone towards EDTA disc was considered positive. Combination disc test was done with Ertapenem and Ertapenem with EDTA. An increase in zone diameter with combination disc of more than or equal to $5 \mathrm{~mm}$ was considered as positive. Clover leaf pattern on the Muller Hinton plate with in a lawn ATCC Escherichia coli and test strain streaked perpendicular to it indicates positivity. Carba $\mathrm{Np}$ test was interpreted as positive if the test strain turns orange to yellow colour on incubation with carbapenems and phenol red as indicator.

Carba Np test is found to be the most specific and sensitive method to detect carbapenamases production. In our study also we found that Carba $\mathrm{Np}$ test is most specific $(100 \%)$ and sensitive (100\%) in detecting carbapenamases and it was better than Modified Hodge test ( sensitivity 91\% specificity $100 \%$ ),Combination disc test (sensitivity $92 \%$ and specificity $50 \%$ ) and Double disc synergy test (sensitivity $42 \%$ and specificity $50 \%$ ).

In another study on comparison of various phenotypic tests (DDST, CDT, MHT). Modified Hodge test was comparatively more specific and sensitive than Double disc synergy test and combination disc test ${ }^{4}$.
Amjad et al., observed carbapenamases in $69 \%$ of isolates (Escherichia coli, Klebsiella pneumoniae, Pseudomonas aeruginosa, Acinetobacter baumanii, Citrobacter diversus, and Enterobacter agglomerans) by Modified Hodge test ${ }^{5}$.

In a study on comparison of carbapenemase detection using a novel chromogenic rapid test-the Carba NP test and the modified Hodge test (MHT).Sensitivities were found to be comparable (CNP, 100\%, versus MHT, 98\%; P 0.08), but Carba $\mathrm{Np}$ was more specific $(100 \%$ versus $80 \%$; $\mathrm{P}<0.0001)$ and faster ${ }^{6}$.

In a similar study Carba NP test detected all carbapenemase producers regardless of the type of the carbapenemase and of their mucoid phenotype with $100 \%$ sensitivity and $100 \%$ specificity ${ }^{7}$.

In conclusion the indiscriminate use of antibiotics led to the development of drug resistance to almost all antibiotics discovered. The development of carbapenem resistance especially in the members of Enterobacteriace, which are the commonest etiological agents in various infections. Being a plasmid coded drug resistance it is spreading with enormous speed not only within the country but also in various nations across the globe. Appropriate detection of such emerging mechanism of resistance goes in along way in preventing unnecessary mortality and morbidity among patients. Effective faster and easier methods like carba $\mathrm{Np}$ test helps us in earlier detection of these infections.

\section{Acknowledgement}

I sincerely thank Dr S. Ramalingam and Dr B. Appalaraju for permitting me to carry out this work in the Department of Microbiology PSGIMS\&R Coimbatore. I acknowledge the 
help rendered by my supervisor Dr.B.Appalaraju for guiding me at all levels.

\section{References}

1. Tille, Patrica. Bailey and Scott's diagnostic Microbiology. Elsevier Health sciences, 2013.13 edition p308-310.

2. Longo, Dan, Anthony Fauci, Dennis Kasper and Stephen Hauser. Harrison's principles of Internal medicine $18^{\text {th }}$ edition. McGraw-hill professional, 2011, $18^{\text {th }}$ edition $\mathrm{p} 1251-54$.

3. Queenan AM, Bush K. Carbapenemases: the versatile $\beta$-lactamases. Clin Microbiol Rev. 2007;20:440-58

4. Balan, K., P. Sireesha, and C. R. Setty. "Study to detect incidence of carbapenemase among Gram negative clinical isolates from tertiary care hospital." J of Dental and Medical Science 1, no. 6 (2012): 8-12.

5. Amjad A, Mirza I, Abbasi S, Farwa U,
Malik N, Zia F. Modified Hodge test: A simple and effective test for detection of carbapenemase production. Iranian Journal of Microbiology. 2011; 3(4): 189193.

6. Vasoo, Shawn, et al. "Comparison of a novel, rapid chromogenic biochemical assay, the Carba NP test, with the modified Hodge test for detection of carbapenemase-producing Gram-negative bacilli." Journal of Clinical Microbiology, 51.9 (2013): 3097-3101.

7. Dortet, L., Poirel, L., and Nordmann, P. (2014). Further Proofs of Concept for the Carba NP Test. Antimicrobial Agents and Chemotherapy, 58(2), 1269. doi:10.1128/AAC.01825-13.

8. Sanger, Frederick, Steven Nicklen, and Alan R. Coulson. "DNA sequencing with chain-terminating inhibitors." Proceedings of the National Academy of Sciences 74, no. 12 (1977): 5463-5467.

\section{How to cite this article:}

Uma Maheswari, M. 2020. Comparison of Phenotypic Methods of Detection of Carbapenamases in a Tertiary Care Hospital, South India. Int.J.Curr.Microbiol.App.Sci. 9(12): 183-188. doi: https://doi.org/10.20546/ijcmas.2020.912.025 